\title{
La quête d'un sens dans l'écriture de soi chez Nawal El-Saadawi et Simone de Beauvoir
}

\section{Ramadan Hemdan Mohamed}

\author{
Département de la langue et de littératures françaises \\ Faculté des lettres, Université de Hélouân
}

\section{Résumé concis}

Notre étude concerne l'expression de la condition humaine, y compris la quête d'un sens dans un monde qui perd le sens, un monde dominé par l'absurde et condamné par la mort, qui cause une angoisse existentielle profonde à l'homme. Nawal El-Saadawi et Simone de Beauvoir tentent de se débarrasser de cette souffrance en retournant au passé, en cherchant le paradis perdu, en réinterprétant et en reconstruisant le passé dans la quête du sens de cette existence. Ce travail s'intéresse à l'examen des idées en mettant en parallèle la vision de chacune des deux écrivaines.

La quête d'un sens est considérée comme acte de révolte existentielle contre la condition humaine, autrement dit contre l'absurdité d'un monde qui perd un sens. L'écriture de soi est aussi une lutte contre le destin dans une tentative de perpétuer l'existence humaine en retirant les souvenirs des griffes du passé et en résistant à leur enterrement dans le cimetière de l'oubli. L'écriture de soi constitue une volonté ensevelie chez l'écrivain dans sa tentative permanente pour l'immortalité après la mort. Si l'écrivain accepte l'annihilation physique, il rejette l'anéantissement moral et l'angoisse et la douleur qu'il porte. Nous trouvons cet aspect de révolte chez les deux écrivaines.

\section{Mots clés}

\section{Révolte ; quête ; condition humaine ; souffrance ; absurde}

Ce travail de recherche traite le thème de la quête d'un sens dans l'écriture de soi ou bien l'autobiographie chez l'écrivaine égyptienne, Nawal El-Saadawi et l'écrivaine française, Simone de Beauvoir. Les deux écrivaines ont un impact significatif sur la réalité culturelle, sociale et littéraire parce qu'elles ont abordé des sujets tabous dans leur société et parce qu'elles possèdent une énorme culture et une grande expérience. Nawal El-Saadawi a travaillé comme pneumologue puis psychiatre et elle est une activiste politique marxiste et une féministe passionnée de littérature. Quant à Simone de Beauvoir, elle était professeure de philosophie et elle était considérée comme activiste féministe, politique et 
existentialiste, elle a joué un rôle très important dans les milieux intellectuels, elle a noué des relations avec de grands écrivains et des philosophes très célèbres, elle a accompagné le philosophe existentialiste Jean-Paul Sartre jusqu'à sa mort.

Ce travail de recherche fait partie de la littérature comparée que Claude Pichois et André-Michel Rousseau, dans son livre, La littérature comparée ${ }^{1}$, ont définie comme :

"La littérature comparée est l'art méthodique, par la recherche de liens d'analogie, de parenté et d'influence, de rapprocher la littérature d'autres domaines de l'expression ou de la connaissance, ou bien les faits et textes littéraires entre eux, distants ou non dans le temps ou dans l'espace, pourvu qu'ils appartiennent à plusieurs langues ou plusieurs cultures, fissent-elles décrire les parties d'une même tradition, afin de mieux les comprendre et les goûter. " ${ }^{2}$

D'après la définition précédente, on va procéder à une méthodologie thématique s'appuyant sur les ressemblances et les divergences chez les deux écrivaines, cette étude va examiner quelques aspects de leur vie qui ont agi sur leur pensée, on va opter pour une comparaison en simultanée en menant une investigation à la fois sur le plan social et l'autre intellectuel.

Selon Etiemble dans son livre, la discipline de la littérature comparée se relie à l'humanisme Comparaison n'est pas raison, ${ }^{3}$ le Congrès de Budapest, l'association internationale de littérature comparée, a affirmé que la production intellectuelle et celle matérielle se constituent d'une interdépendance universelle des nations, selon Karl Marx, la littérature universelle n'est qu'un produit de nombreuses littératures nationales et même provinciales. Montesquieu a refusé toute littérature qui ne s'est pas intéressée au genre humain, il l'a regardée comme un crime, l'universalité de la littérature ne s'est pas limitée seulement à traiter les relations entre les différentes littératures d'une seule époque, mais aussi elle doit étudier l'histoire de ces relations, il s'agit d'une étude synchronique et diachronique.

${ }^{1}$ Titre d'un livre, PICHOIS (Claude) et ROUSSEAU (André-Michel), La littérature comparée, Paris, Armand Colin, 1967.

2 https://theses.univ-

lyon2.fr/documents/getpart.php?id=lyon2.2006.cisse m\&part=109998

${ }^{3}$ Titre d'un ouvrage, ETIEMBLE, Comparaison n'est pas raison, Paris Gallimard, 1963. 
Nawal El-Saadawi et Simone de Beauvoir ont abordé presque les mêmes problèmes de l'homme en général et des femmes en particuliers à tel point que certains disent que Nawal El-Saadawi est Simone de Beauvoir des arabes.

Le titre de notre étude provoque une problématique : y a-t-il un rapport entre l'écriture de soi et le sens ? Que veut dire le mot «quête »? les deux écrivaines ont-elles eu un sens définitif de leur vie?

Notre travail va traiter d'abord le contexte social et culturel en Egypte et en France pour que nous puissions connaitre les facteurs qui ont contribué à la formation intellectuelle des deux écrivaines, nous allons ensuite examiner l'autobiographie ou l'écriture de soi d'après Philippe Lejeune et la révolte selon Julia Kristeva en vue de découvrir la relation entre les deux notions. Après la partie socioculturelle et l'autre définitionnelle, nous allons attaquer le thème principal de notre étude désignée par le titre. Enfin, nous allons passer en revue dans la conclusion les aboutissements et les résultats de cette recherche.

\section{Un aperçu sur les conditions socioculturelles et le féminisme.}

Les deux écrivaines sont considérées comme des pionnières du féminisme, c'est pour cela, il faut aborder un aperçu sur ce mouvement et son évolution en faisant la lumière sur les conditions socioculturelles en Egypte et en France,

Dans la société traditionnelle égyptienne, la norme impose la réclusion des femmes et la domination masculine sur les espaces publics. Entre 1769 et 1849 , lors du règne de la dynastie des Mohamed Ali, on a témoigné de grandes réformes dans plusieurs domaines y compris la fin des harems. Rifa El-Tahtawi, Mohammad Abdu ont prôné dans leurs écrits l'instruction des femmes, Mohamed Abdu a demandé une stricte limitation de la polygamie. En outre, Qasim Amîn a condamné la réclusion et la claustration absolue des femmes dans les harems. Il a appelé l'allègement de la séparation entre les sexes dans les espaces publics, il a également revendiqué la limitation de la polygamie, l'arbitrage du divorce par un juge et l'accès des filles à l'instruction au même titre que les garçons :

La presse égyptienne a joué un rôle majeur dans la question féminine grâce à une communauté syrienne qui a fondé en Egypte les premiers journaux de femmes, on peut citer des femmes comme Warda Al-Yazigi, Maryam Al-Nahas, Zaynab Fawaz, des journaux comme al-fatat (la Jeune fille) créé en 1892 par Hind Nawfal, anîs al-jalîs (le Compagnon intime) en 1898 par Alexandra Khouri Avierino ; Fatat Al-sharq (la Jeune fille d'Orient) en 1906 par Labîba Hachem. 
Ces journaux ont donné la chance aux femmes de provoquer des débats sur l'accès des femmes à l'éducation, la mixite, les conditions de mariage, de divorce.

Par ailleurs, les salons littéraires ont constitué un espace de défendre la cause féminine, on peut mentionner le salon de May Zyada tenu dès 1911, ce salon hebdomadaire accueillent des hommes politiques, des poètes, des journalistes ainsi que des écrivains célèbres comme Khalîl Mutrân, Lutfî asSayyid, Taha Hussein.

Le contexte de l'entre-deux guerres a permis aux femmes de prendre part à la vie publique, d'accéder à l'espace public, à l'instruction et au salariat. Elles ont participé à la manifestation de la révolution de 1919 de Saad Zaghloul contre l'occupation britannique. Parmi elles, il y a eu Huda Sha'rawi et Saiza Nabarawi. On remarque des inégalités socioculturelles au sein de la société égyptienne, la situation des femmes en ville diffère de celle des femmes à la campagne, la femme rurale est plus enfermée que celle citadine. A partir de 1919, on observe l'affirmation des mouvements des femmes et l'émergence d'un mouvement féministe égyptien, à titre d'exemple, Huda Sharawi a représenté le symbole du féminisme égyptien, elle a été présidente du comité du parti Wafd pour les femmes, Saiza Nabarawi et elle ont fondé l'union féministe égyptienne en 1923 et beaucoup de centres médico-sociaux dédiés aux femmes pour militer contre l'analphabétisme, la pauvreté et les maladies dont sont victimes les femmes, Huda Sharawi a également créé deux journaux en français (l'Egyptienne) en 1925 puis en arabe (Al-Masriyya) en 1937. On peut citer d'autres figures : Malak Hifni Nasef connu par son recueil d'articles et de discours sur les femmes et Zaynab Fawwaz connue également par son recueil d'articles Al-rassâ'il Al-zaynabiyya (Les correspondances de Zaynab) dont le thème se rapportait souvent aux droits et à la place des femmes dans la société.

En 1911, Malak Hifni Nasef avait demandé, au Congrès égyptien, des améliorations de la situation des femmes égyptiennes dans le travail, l'âge minimum au mariage, le divorce... Zaynab Al-Ghazali qui fait partie du courant rigoriste « le féminisme islamique », a créé en 1936 « l'association de la femme musulmane ». À partir des années 20, Nabawiyya Musa a revendiqué dans son livre Al-mar'a wal- 'amal (La femme et le travail) le droit des femmes au travail. Cependant, cette question n'a été posée clairement que lors de la création, au Caire, de la Fédération féministe arabe en 1938. Durriya Shafiq a publié la célèbre revue Bint aL-nil (Fille du Nil) en 1945, elle a créé une association du même nom qui est devenue un parti politique en 1948, le premier parti féministe en Égypte. 
Elle a mené plusieurs manifestations réprimées sous le règne de Gamal Abdel Nasser, on l'a condamnée au silence et à l'emprisonnement à vie, mais Durriya Shafiq s'est suicidée en 1975. Les femmes égyptiennes n'ont obtenu le droit de vote qu'en 1956. Le parlement égyptien a reçu en 1957 la première femme égyptienne élue Rawya 'Atiyya,

Le règne du président Gamal Abdel Nasser a témoigné de l'interdiction des associations de femmes, ces dernières se sont orientées vers les œuvres caritatives. Dans les années 70, le féminisme spontané était absente en faveur du féminisme de l'État, selon la constitution de 1971, l'égalité des sexes a dépendu des dispositions de la shari'a islamique, cet acte a empêché les femmes de participer au travail et à la vie publique et politique sur un pied d'égalité avec les hommes. Le code du statut personnel a décrété la soumission des femmes à l'autorité du père et de l'époux, il s'agissait de légitimer et institutionnaliser l'inégalité entre les sexes. Nawal El-Saadawi a consacré toute sa vie pour défendre les droits des femmes en critiquant les pratiques contre elles comme la question de l'excision des filles, elle a fondé en 1982 une nouvelle organisation féministe Arab Women's Solidarity Association (Association de solidarité des femmes arabes, AWSA), association non gouvernementale et indépendante qui a eu le soutien de plusieurs intellectuels et écrivains ainsi que la reconnaissance des Nations-Unies. Nawal El-Saadawi a publié en 1989 un journal féministe Nun.

Nawal El-Saadawi qui est née en 1931, a peint une image de sa vie heureuse dans la famille et une autre déplorable du village même avant sa naissance : selon elle, le paysan est la personne dont l'urine se mêle au sang, c'est à dire il souffre de la pauvreté, l'ignorance et la maladie, ces trois maux constituent son destin inévitable tandis que les femmes subissaient l'oppression à tous les niveaux et sous toutes les formes. Quand elle s'est déplacée au Caire dont elle a analysé la scène sociale et politique basée sur la dichotomie entre les riches et les pauvres d'une part, entre les partisans du régime politique et les opposants d'autre part. l'Egypte vivait l'époque de féodalité où les grands propriétaires du village possédaient $98 \%$ des terres tandis que $80 \%$ des paysans s'appropriaient $2 \%$ des terres. Nawal El-Saadawi a souligné les événements politiques en Egypte, elle a reflété la société égyptienne à la fois dans les villes et dans les villages en critiquant le régime en cours, d'après elle, elle a critiqué le journal Al-Ahram parce que ce journal faisait l'éloge du roi et conservait l'héritage méprisable, il constituait un des piliers du pouvoir en Egypte, un des instruments d'opprimer le peuple et les esclaves, le slogan de ce journal porte le signe de l'esclavage parce 
que les pyramides ont été construites par les esclaves qui portèrent les pierres sur le dos pour bâtir la tombe du pharaon. Selon Nawal El-Saadawi, dans son livre, Qadaya Al Mara'a walfekr walsiassa (les affaires de la femme, de la pensée et de la politique $)^{1}$, la libération des femmes se lie à leur participation à cette action et au degré de leur conscience de l'importance de cette participation, l'augmentation de leur conscience dépend de plusieurs facteurs : l'extension de l'éducation et du travail des femmes, la participation aux différents métiers, à la politique, à l'économie et à la culture, la prise des responsabilités dans les différents niveaux, l'exercice de la prise des décisions dans l'Etat et à la famille, la participation à la pensée, à la littérature, à l'écriture, aux médias, aux recherches scientifiques et sociales ...etc.

En France, au début du XX $\mathrm{X}^{\mathrm{e}}$ siècle, le statut des femmes est dominé par le Code civil de 1804 qui a stipulé l'incapacité juridique de la femme, les femmes n'ont pas pu joindre les lycées et les universités, elles n'ont pas eu le droit de signer un contrat, de gérer des biens, de travailler sans l'autorisation de leur mari, de voyager sans autorisation, etc.

Le code pénal de 1810 a exercé le même despotisme sur les femmes : l'adultère était puni d'une peine de prison ; le devoir conjugal a constitué une obligation c'est à dire il n'existait pas de viol entre époux, l'avortement était réprimé durement.

Les femmes étaient privées de l'exercice de la citoyenneté sous prétexte qu'elles n'ont pas eu la capacité de comprendre les enjeux politiques, que plusieurs facteurs influencent la pensée des femmes comme leurs émotions, le clergé, en conclusion : «les femmes appartiennent à la famille, non à la société politique, et la nature les a faites pour les soins domestiques et non pour les fonctions publiques. $»^{2}$ dit Bonald, un penseur réactionnaire.

Selon les lois Ferry de 1882, l'école primaire est devenue obligatoire pour les jeunes filles âgées de 6 à 13 ans, mais il y avait une différence entre l'éducation des filles et celle des garçons, elles apprenaient les travaux domestiques, la cuisine, etc. Au début du XX ${ }^{\mathrm{e}}$ siècle, une petite minorité des filles était scolarisée dans l'enseignement.

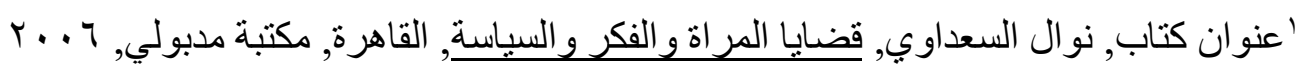

2 BEAUVOIR (Simone de), Le deuxième Sexe I, les faits et les mythes, Paris, Gallimard, 1976, p, 150. 
Il est à noter que Leon Richier qui fut le véritable fondateur du féminisme pour plusieurs raisons ; la création des « Droits de la Femme » en 1869 et l'organisation du Congrès international du Droit des femmes qui s'est tenu en 1878.

$\mathrm{Au}$ début du $\mathrm{XX}^{\mathrm{e}}$ siècle, on remarque l'émergence des premiers mouvements féministes radicaux en France, par exemple, Madeleine Pelletier, médecin-psychiatre, militante d'extrême gauche, elle a encouragé la contraception et pratique l'avortement. La plupart des premières féministes ne revendiquaient que le droit de vote.

Pendant la première Guerre mondiale, les femmes ont remplacé les hommes dans les champs, les usines (les “munitionnettes"), les hôpitaux, l'assistance sanitaire auprès des enfants, elles administraient le budget dans les foyers.

Après la fin de la guerre, on a rappelé les femmes à leur rôle d'épouses et de mères à cause de la saignée démographique qui a entrainé les lois natalistes de 1920 et 1923, selon ces lois, on a infligé une peine à toute propagande pour la contraception et l'avortement était puni d'une peine de mort.

Dans les années 1920, les mouvements qui ont demandé le droit de vote pour les femmes se sont répandus. Dans les années 1930, Louise Weiss et son association " La femme nouvelle " ont fait plusieurs manifestations en faveur du droit de vote des femmes.

Le général de Gaulle et le GPRF (Gouvernement provisoire de la République française) ont octroyé aux femmes le droit de vote et d'éligibilité (21 avril 1944). Le préambule de la constitution de 1946 et celle de 1958 ont approuvé le principe d'égalité. Après l'égalité, les féministes ont commencé à demander la parité surtout dans le domaine politique. De même, on a constaté après la $2^{\mathrm{e}}$ guerre mondiale que la proportion de femmes dans la population active augmente (avec 13,3 millions d'actives pour 14,7 millions d'hommes, on a approché de la parité).

\section{Le rapport de l'écriture de soi à la révolte.}

Julia Kristeva a examiné dans son livre, Sens et non-sens de la révolte, pouvoirs et limites de la psychanalyse 1, le thème de la révolte dans ses différentes significations à travers les époques et les cultures,

sous l'influence de l'italien, aux $X V^{e}$ et $X V I^{e}$ siècles, volutus, voluta- en français, "volute ", comme terme d'architecture - de même que volta et voltare 
suggèrent l'idée d'un mouvement circulaire et par extension, d'un retour temporel. Volta signifie aussi "fois "d'où "retournement"1.

Cette étude prend pour genre littéraire l'autobiographie que Philippe Lejeune a définie comme :

" un récit rétrospectif en prose qu'une personne réelle fait de sa propre existence, lorsqu'elle met l'accent sur sa vie individuelle, en particulier sur l'histoire de sa personnalité.",2

Les deux définitions nous conduisent à découvrir une ressemblance entre l'écriture de soi ou l'autobiographie d'un côté et la révolte d'un autre côté. Tous les deux concepts s'intéressent au concept du retour sur soi, ils tentent de retrouver le soi complet à travers la remémoration et la narration.

Jean Philippe Miraux a analysé cette définition dans son livre, l'autobiographie, écriture de soi et sincérité, il a souligné quatre spécificités de ce genre : la première caractéristique est la forme du langage qui désigne un récit en prose ; la deuxième, le sujet traité qui a pour objet une vie individuelle et une histoire d'une personnalité ; la troisième, la situation de l'auteur qui signifie l'identité de l'auteur et du narrateur, il s'agit que le nom renvoie à une personne réelle ; la quatrième, la position du narrateur qui indique l'identité du narrateur et du personnage principal d'une part, la perspective rétrospective du récit d'autre part, il est à noter que les deux dernières spécificités constituent pour Philippe Lejeune les deux conditions incontournables. Le signe de l'identité entre le narrateur et le personnage principal est l'emploi de la première personne, il s'agit d'une narration autodiégétique selon l'appellation de Gérard Genette dans son livre Figures III $^{3}$. Il existe un pacte autobiographique qui signifie l'affirmation de l'identité du nom (auteur- narrateur- personnage) dans le texte. Ce pacte définit l'attitude du lecteur, si l'identité n'est pas affirmée (cas de la fiction), il cherche les ressemblances tandis que si elle est affirmée (cas de l'autobiographie), il cherche les différences (erreurs, déformations) et les ruptures du contrat.

Les œuvres autobiographiques des deux écrivaines ont répondu aux conditions du genre, elles ont été écrites en prose, Nawal El-Saadawi et Simone

\footnotetext{
${ }^{1}$ KRISTEVA (Julia), Sens et non-sens de la révolte, pouvoirs et limites de la psychanalyse 1, Fayard, France, 1996, p.9

${ }^{2}$ LEJEUNE (Philippe), Le pacte autobiographique, Paris, Seuil, 1975, p. 14.

3 Titre d'un livre, GENETTE (Gerard), Figures III. Paris VI ${ }^{\mathrm{e}}$, Seuil, 1987. 
de Beauvoir ont eu pour thème leur vie personnelle, on trouve l'identité entre l'auteur et le narrateur d'un côté, entre le narrateur et le personnage principal d'un autre côté, c'est à dire qu'elles ont signé leur pacte autobiographique avec le lecteur en donnant leur nom et en utilisant le premier pronom personnel du sujet « je ».

Dans le récit autobiographique, la présence du nom de l'auteur désigne par une convention sociale l'engagement de responsabilité d'une personne réelle, le lecteur n'a pas besoin de vérifier l'auteur même s'il ne le connait pas, son existence est indubitable. L'auteur se trouve à cheval sur le hors-texte et le texte. Les techniques de l'autobiographie possèdent en tant qu'aventure personnelle, des significations : l'expression de l'éthique d'une culture; l'expression de la vie ou de l'univers de soi ; l'expression du jugement, le refus et l'acceptation. Bref, la technique de l'écriture n'est jamais gratuite, elle renvoie à une signification psychologique de l'attitude de l'écrivain ou de celle de toute une culture, Nawal El-Saadawi décrit l'époque dans le monde d'une femme, elle considère son époque comme entrée dans son univers individuel qui occupe la place primordiale dans son autobiographie, autrement dit les œuvres de Nawal El-Saadawi sont marquées par une volonté de contempler son image sur la page blanche tout en sacrifiant la description objective de l'époque. Sa révolte est plutôt une révolution presque violente. Elle veut tout détruire pour reconstruire un autre monde exclusivement féminin. Par contre, Simone de Beauvoir se raconte en tant que femme tout en se situant dans l'époque, c'est à dire, elle recule en donnant le devant de la scène à la description de son époque et à la condition féminine liée à l'histoire. Elle voulait changer le monde par la réforme.

Les autobiographes essaient en général d'attirer la pitié en présentant un argument ad misericordiam, ils mettent en évidence leur souffrance avec grandiloquence et ont recours à l'emphase pour atteindre un amour d'un public plus large, cette souffrance leur représente une source de connaissance, une marque d'élection et un moyen de parvenir à une lucidité décisive, ils considèrent leur vie comme une longue souffrance.

Nawal El-Saadawi a suivi une technique très claire : c'est celle du traditionnel discours autobiographique, la narration a occupé plus de place que la description, l'écrivaine égyptienne a reflété sa subjectivité en s'intervenant énormément dans son œuvre à chaque moment, en commentant chaque évènement, en avouant ses opinions, c'est pourquoi elle s'est déplacée, toujours entre le présent et le passé, elle a mélangé les moments présents à ceux du passé 
comme s'ils étaient un seul moment, elle voulait donner son interprétation. Par exemple, le titre du premier chapitre du premier volume Aoraki Hayati, Ainsi je suis venue au monde ${ }^{1}$ où elle a pris comme approche sa naissance pour parler de la haine sociale et historique contre les filles surtout à la campagne parce que les familles s'attristaient de la naissance des filles en même temps qu'elles avaient la joie d'avoir les garçons. Pour Nawal El-Saadawi, cette hostilité contre les femmes remonte à la culture préislamique quand on ensevelissait les nouveau-nées en vie.

Par contre, Simone de Beauvoir a suivi dans Mémoires d'une jeune fille rangée une technique d'écriture particulière, elle s'est appuyée sur la contingence et la facticité comme dans le roman, elle était en continuelle recherche d'un sens car l'absence de raison de vivre lui était d'autant plus affreux que de mourir. Elle choisit le fait de s'en tenir à un pur récit, elle a eu recours plus à la description que à la narration :

«Par exemple Simone de Beauvoir, dans les Mémoires d'une jeune fille rangée, loin d'afficher la subjectivité liée au récit autobiographique, a l'air de raconter les choses "telles qu'elles furent. $»^{2}$

Simone de Beauvoir n'a éprouvé ni douleur, ni souffrance, ni jalousie pendant son enfance à cause de son sexe. Elle avait seulement une petite sœur.

Mais, l'auteure française annonce dans les volumes suivants le pacte en bonne et due forme, elle développe le traditionnel discours autobiographique fondé sur l'interprétation et la subjectivité, c'est à dire, elle a préféré la narration à la description.

Par ailleurs, l'intimiste égyptienne a priorisé l'ordre thématique dans toutes ses œuvres autobiographiques Aoraki Hayati, Mémoires de la prison des femmes et Rahalaty fi al-alam, c'est à dire, chaque chapitre a porté un titre reflétant son idée principale, chaque titre n'a rien à voir avec les étapes de sa vie, au contraire elle se concentre sur une action qui a influencé sa formation dans chaque phase, cela n'empêche pas qu'elle ait suivi l'ordre chronologique. Nawal El-Saadawi a beaucoup souligné sa douleur dans la société égyptienne soit à la campagne soit en ville à cause de sa féminité d'abord, de sa pensée ensuite, lors de son enfance à la campagne, son maître d'école a étouffé son talent dans l'écriture lorsqu'il a lu son premier roman, mais ses parents admiraient son adresse, les maîtres ne

1 Texte intégral :

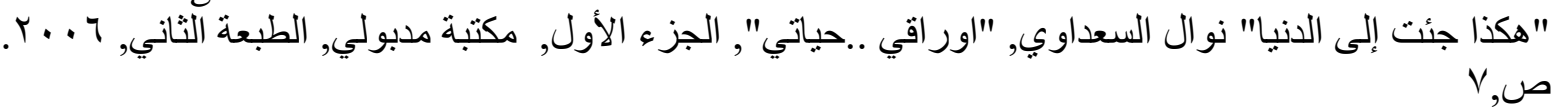

${ }^{2}$ LEJEUNE (Philippe), L'autobiographie en France, Armand Colin, 2004, p. 54. 
supportaient pas ses questions, malgré cela, elle aimait l'école interne où elle pouvait se réjouir premièrement du calme loin des problèmes familiaux pendant son séjour chez son oncle, deuxièmement du jeu avec les filles, elle a envisagé plein de difficultés à cause de ses idées, pour elle, la patrie s'est transformée en lieu où les policiers la poursuivaient: "je suis née une femme dans un monde qui ne veut que les mâles "',

Mais, elle a ressenti son attachement à sa patrie lorsqu'elle enseignait le thème de la révolte et de la création à l'université de Durheim aux Etats-Unis :

' c'est pour la première fois, je connais le sens de la patrie, l'amour de la patrie nait comme une forte cataracte qui brise les barrières entre le rêve et la vérité ..., ",

L'écrivaine égyptienne avait un projet qui vise réinterpréter son passé, elle était plus liée à son enfance que Simone de Beauvoir, elle n'avait aucun problème avec cette phase de sa vie, elle la voyait comme un paradis perdu puisqu'elle n'était rusée par aucune valeur bourgeoise, elle a témoigné ses douleurs propres, celles des autres femmes et des pauvres notamment à la campagne à cause de cette idéologie qu'elle détestait, elle s'aidait plus de narration que la description dans ses écrits autobiographiques, on entend la voix adulte partout.

Quant à Simone de Beauvoir, elle a combiné l'ordre chronologique et l'autre thématique dans Mémoires d'une jeune fille rangée, cette œuvre se compose de quatre parties : dans la première partie, l'intimiste française a observé l'ordre chronologique, dans la deuxième, elle a amalgamé l'ordre chronologique et celui thématique, la troisième et la quatrième ont concentré sur celui thématique. Ainsi son écrit autobiographique diffère d'autres autobiographies qui portent sur le récit d'enfance reflétant un attendrissement attendu parce que Simone de Beauvoir avait un projet qui consiste dans l'objectivité de son récit et l'unité romanesque de sa première autobiographie.

Philippe Lejeune a défini les deux buts de chaque autobiographie : communiquer avec son passé et se dévoiler à autrui (le lecteur).

1 Texte intégral :

"لقد ولدت انثي في عالم لايريد إلا الذكور" نوال السعداوي, "أور اقي ..حياتي", الجزء الأول. نفس المرجع, ص.

${ }^{2}$ Le texte intégral

" لأول مرة اعرف معنى الوطن, يولد في الحب شلالا هادرا يكتسح الحواجز بين الحلم والحقيقة... " نوال 
Les autobiographies de Nawal El-Saadawi et de Simone de Beauvoir retracent les changements qui affectent leurs auteures, elles ressemblent au roman d'apprentissage parce que les deux écrivaines y ont montré leur cheminement évolutif où elles ont suivi le développement de leur existence en arrivant en fin de compte au sens de la vie. Elles ont avoué une conception de la vie qu'elles se sont forgées.

Lejeune a également indiqué que la rhétorique de l'autobiographie prend deux directions : l'inavouable et l'ineffable:

"L'inavouable signifie tout dire, et en particulier ce dont on ne parle guère à autrui : la sexualité. [...] l'ineffable : si l'auteur fait confiance au lecteur et lui laisse imaginer ces réalités sexuelles qu'il n'évoque qu'à mots couverts.il désespère au contraire de jamais lui faire comprendre les minutes de poésie ou de révélation: ces moments mystérieux et uniques, dont il sent la vibration en lui, sont au-delà de tout langage ». ${ }^{1}$

Selon Philippe Lejeune, l'ineffable constitue un sentiment qui apparaît clairement dans l'expérience religieuse de l'auteur.

Nawal El-Saadawi n'a pas parlé de sa libido sexuelle, elle se considère comme un être surnaturel, c'est-à-dire, elle ne se soumet jamais aux instincts sexuels comme tous les êtres humains. Elle se voit la déesse Isis et Jeanne d'arc, Il est à noter qu'elle a joué le rôle de la déesse Isis au théâtre de son école primaire, elle a répété cette action en affirmant que l'esprit divin de cette déesse habite son corps, qu'il la protège de toute inquiétude. De même, elle imaginait, dans ses rêveries, qu'elle luttait comme Jeanne d'arc contre l'occupation britannique en cours et qu'elle a pu libérer sa patrie :

« Je ne m'imaginais pas comme médecin qui tient une longue seringue et qui l'injecte dans les bras des gens, je me voyais assise devant un piano pour jouer de la musique, je chante et danse, je marche par terre en portant à la tête le disque du soleil comme la déesse Isis. ${ }^{2}$

C'est pourquoi, elle a avoué qu'elle pensait à un être incomparable :

"La nuit, je rêve de lui, le matin, je suis à la cherche de lui, d'elle, d'eux ou d'elles, cet être dépasse tous ces classements, il est fait en matière qui n'est ni

${ }^{1}$ L'autobiographie en France. ibid, pp. 53, 54

2 Texte intégral :

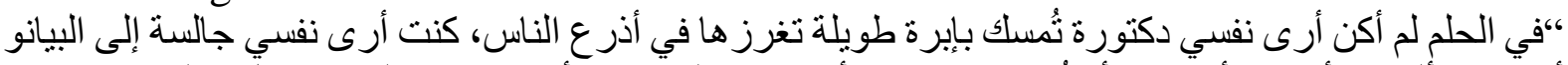

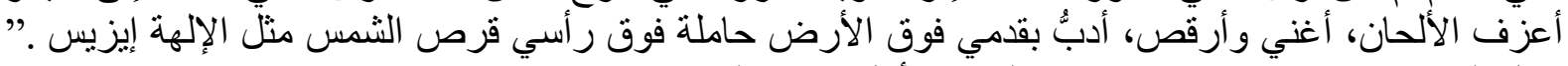

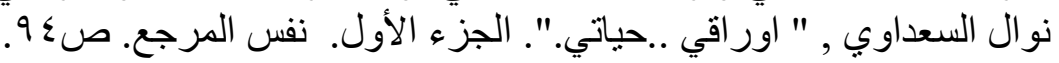


l'âme, ni le corps, ni l'esprit, cette matière confond les trois éléments en une nouvelle composition incomparable, cet être n'a ni barbe, ni moustache, ni seins, ni derrière, ni utérus, ni organe masculin. Néanmoins, il possède les traits de l'être humain. »"

Au sujet de l'ineffable, l'autobiographe égyptienne a indiqué plusieurs fois que le fait d'écrire n'est pas capable d'exprimer ses sentiments, par conséquent, elle quittait la plume en vue de se débarrasser de cet état pénible.

A propos de l'inavouable chez Simone de Beauvoir, celle-ci n'a parlé de la sexualité qu'une seule fois lors de son enfance : «Pour m'absoudre, il posait sur ma nuque sa main de justicier : implorant son pardon, j'obtenais la volupté [...] Pour de vrai, je ne me soumettais à personne : j'étais, et je demeurerais toujours mon propre maître. $»^{2}$ En ce qui concerne l'ineffable ou bien les moments mystiques, cet aspect n'apparaissait guère dans ses œuvres autobiographiques, ce manque remonte à son athéisme et à sa conviction de la théorie existentialiste.

\section{Le sens de la vie chez les deux écrivaines}

Les autobiographes en général veulent toujours présenter dans leur écriture de soi, un sens à leur vie, qu'est-ce que c'est qu'un sens? Le dictionnaire Le petit Robert $^{3}$ indique plusieurs acceptions y compris : une signification, une valeur et une direction. Ces sens précédents ressemblent aux acceptions de la révolte en tant qu'une interprétation et une évolution.

Philippe Miraux a montré dans son livre, L'autobiographie, écriture de soi et sincérité, les opinions de certains écrivains sur l'écriture intime, Chateaubriand affirme que l'écriture de notre existence permet de saisir la signification de la totalité de la vie. Pour Georges Bataille, il commente les peintures de Lascaux :

${ }^{1}$ Texte intégral :

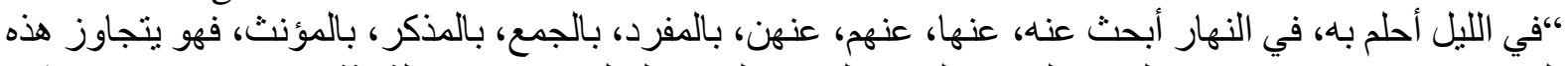

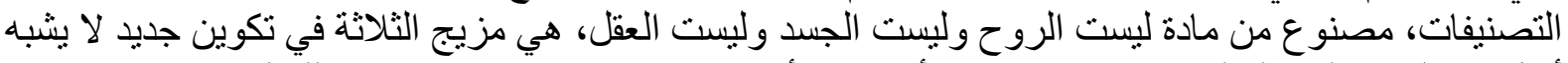

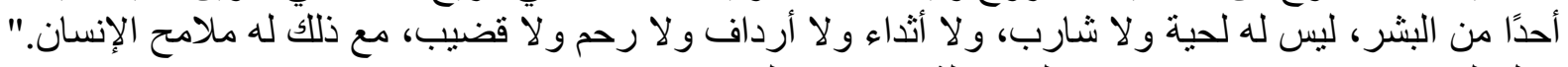

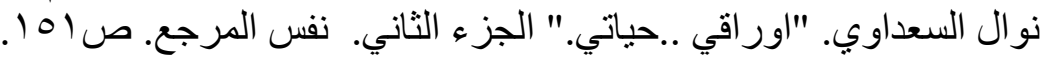

${ }^{2}$ Simone de Beauvoir, Mémoires d'une jeune fille rangée, Paris, Gallimard, 2008, p. 79

${ }^{3}$ Titre d'un dictionnaire, REY (Alain), le dictionnaire le Petit Robert, France, 2014. Journal of Scientific Research in Arts 
«Le geste artistique est, certes, la volonté humaine de saisir la réalité, de figer l'insaisissable, d'expliquer imaginairement et métaphoriquement l'univers. " ${ }^{1}$

Ce statut ne diffère pas de celui de l'autobiographe. Mais celui-ci se trouve devant le désordre et la surabondance des souvenirs, il possède une mémoire caractérisée par la complexité et la richesse que le récit ne peut pas respecter, il doit adopter un ordre soit chronologique soit logique ou thématique : " ce que poursuit l'autobiographie, c'est le sens de la vie. La mémoire est un labyrinthe où l'on cherche le fil d'Ariane. "' 2 Julien Green affirme que la quête d'un sens représente le but de tous les écrits autobiographiques : "L'écriture autobiographique est présentée non comme l'exposition d'un sens déjà connu, mais comme la recherche du sens. $»^{3}$

Nawal El-Saadawi a quêté le sens de la vie dans l'écriture, celle-ci ressemble à l'amour qui se passe sans raison, malgré cela, l'autobiographe égyptienne n'a pas cessé de poser la question à propos de la raison de l'écriture de son autobiographie en donnant plusieurs hypothèses sous la forme de nouvelles questions : la nostalgie au temps passé, sauver les mémoires avant que le temps passe, retenir les images avant de disparaitre, ou lutter contre le néant pour rester à l'être ou l'éternité. Elle a ajouté une autre possibilité, la volonté de dessiner au monde ma vraie image que l'on a remplacée par un autre. Bref, l'écriture autobiographie lui a représenté la seule voie de rechercher le sens de sa vie.

"Je lève la tête du dessus de la feuille, je la laisse un moment, pourquoi j'écris mon autobiographie aujourd'hui ? la nostalgie à l'âge qui est passé? estil passé ? ou y a-t-il un reste? les mots sont-ils le dernier refuge pour saisir ce qui est passé avant de disparaitre ? fixent-ils les images à la mémoire avant le déclin ? résistent-ils à la mort pour exister ou l'immortalité ? ',4

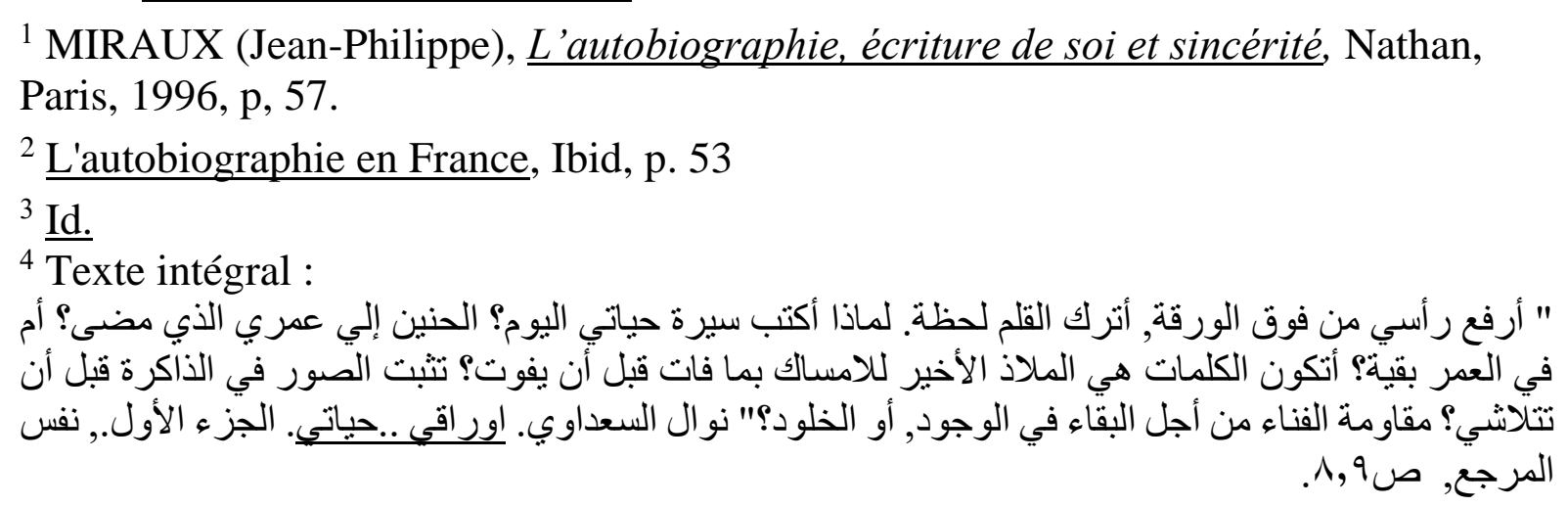


Simone de Beauvoir cherchait continuellement le sens de son existence : "Pourquoi suis-je ici ?" Elle essayait de le trouver dans l'écriture, cette dernière lui représente le moyen de réaliser son rêve, tel était l'absolu qui compense l'éternité perdue pour un cœur épris d'absolu :

“ il n'y avait plus de Dieu pour m'aimer, mais je brûlerais dans des millions des cours ",2

A travers l'écriture, l'écrivaine française voulait parler et communiquer avec le monde et atteindre le sens de son existence, dépasser le non-sens de son existence, elle remporte l'immortalité et prend la place de l'amour divin parce qu'elle est lue et aimée par millions de personnes. Pour elle, le lecteur est le substitut de Dieu, la littérature est comparable à la prière, elle la détache de son milieu, de sa famille et de ses amis pour choisir sa propre vie.

On ne peut pas saisir dès le début les différents sens de la vie chez Nawal El-Saadawi et Simone de Beauvoir, ces dernières changeaient d'un sens à l'autre grâce à la diversité des évènements et des expériences vécus, c'est pourquoi on va découvrir petit à petit les nombreux sens à travers les phases de leur vie. les deux écrivaines sont passées par différents sens, cependant, elles avaient des objectifs communs tels : l'obéissance à la fois à Dieu et à ses parents ; l'ascension ; la révolte, la littérature et la mission d'écrire. Néanmoins, on remarque des détails différents dans ces objectifs chez les deux écrivaines.

\section{3-1- L'obéissance à Dieu et aux parents.}

Pour obéir aux parents, Nawal El-Saadawi excellait à ses études, elle n'attendait que la joie de ses parents qui exerçaient une grande influence sur sa personnalité, elle n'a vécu aucune contradiction entre eux, les deux parents possédaient un caractère révolté, la révolte de sa mère s'adressait aux causes féminines, tandis que celle de son père s'orientait vers les affaires sociales, religieuses et politiques. Mais elle était jalouse de son frère Talaat parce que celuici occupait plus d'intérêt de ses parents que Nawal El-Saadawi, surtout qu'il échouait toujours à l'école, son échec répandait une grande tristesse à la famille et faisait oublier le succès de Nawal El-Saadawi, il s'agit que cette dernière souffrait de la supériorité masculine à l'intérieur de sa famille.

${ }^{1}$ Mémoires d'une jeune fille rangée, Ibid, p. 90.

2 Ibid, p. 187. 
Simone de Beauvoir cherchait à plaire à ses parents par tous les moyens, elle s'intéressait à ses devoirs scolaires, elle achevait ses apprentissages avec succès pour obtenir la satisfaction de leurs parents parce qu'elle était convaincue que ses parents n'entendent que son bien, Son père cultivait l'art et il lui représentait le côté intellectuel tandis que sa mère s'occupait toujours de lui apprendre les préceptes catholiques, elle lui symbolisait l'aspect religieux, Simone de Beauvoir procure la satisfaction du père par le progrès scolaire, celle de la mère par l'apprentissage religieux. Cette attitude lui garantit la tranquillité.

Malgré la ressemblance apparente concernant l'obéissance aux parents, on constate deux points différents sur les conditions familiales : l'harmonie des parents de Nawal El-Saadawi, le désaccord personnel entre les parents de Simone de Beauvoir ; il existait une jalousie chez Nawal El-Saadawi tandis que cet aspect était absent chez Simone de Beauvoir.

\section{3-2- L'ascension}

On constate que le mot « ascension » avait un rapprochement des termes, la direction et l'évolution appartenant à la notion du sens, à celle de la révolte. L'ascension se révélait clairement dans l'éducation chez les deux écrivaines.

Pour Nawal El-Saadawi, elle donnait une grande importance à l'enseignement qui lui incarnait une libération du destin de sa mère et toutes les filles de sa campagne, elle avait la capacité de créer des contes, elle haïssait d'être absente de son école, même si elle était malade, rien ne l'a empêchée d'aller à son école, elle a considéré l'absence de l'école comme la mort, la sortie pour l'école symbolise la libération, l'éloignement de la terre et le rapprochement du ciel. L'école était le seul chemin de se libérer et de retrouver le bonheur. Au cycle secondaire, Nawal El-Saadawi aimait sa maîtresse de l'anglais Mademoiselle Sania, celle-ci racontait des romans anglais, elle avait de bonnes relations avec toutes les élèves avec lesquelles elle discutait, Nawal El-Saadawi voyait que Mademoiselle Sania ressemblait à sa mère, l'autobiographe égyptienne ressentait dans ce monde une solitude dont sa maitresse Sania la sauvait :

"Elle possédait une lumière dans ses yeux, quand elle me voyait, elle me tirait de mon dépaysement dans ce monde, la maussaderie se dissipait, le profond chagrin inconnu s'en allait. ${ }^{1}$

1 Texte intégral :

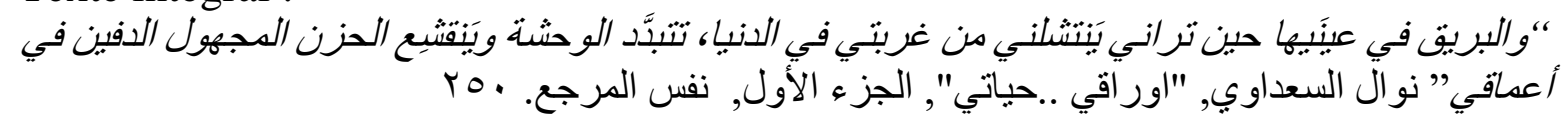


Pour Simone de Beauvoir, cette ascension intellectuelle se traduit par des actions et par ses études, dès son enfance, Simone de Beauvoir affirme qu'elle maîtrise sa vie, qu'elle n'a pas laissé le concept du bio l'emporter sur celui de l'auto: "Les joies et les peines des hommes correspondent à leurs mérites. ",

L'existentialisme sartrien a exercé une grande influence sur Simone de Beauvoir, cette philosophie de l'individu et du projet a une complicité particulière avec la démarche autobiographique. Simone de Beauvoir s'est orientée au passé avec ses théories existentialistes qui existent clairement dans la dernière autobiographie, Tout compte fait qui a donné la signification des mémoires, elle ne s'est attachée ni au passé, ni au sens déjà préétabli, mais elle devait reconquérir le sens de sa vie qui n'est jamais donné d'avance : "déclarer l'existence absurde, c'est nier qu'elle puisse se donner un sens, dire qu'elle est ambiguë, c'est poser que le sens n'en est jamais fixé qu'il doit sans cesse se reconquérir ', 2.

C'est pour cette raison, Simone de Beauvoir était très occupée à se développer, elle tenait sa vie pour une ligne droite dans le sens d'une ascension, elle aimait le travail corporel et intellectuel et elle détestait l'oisiveté, elle voulait exceller en tout :

' Tant de choses m'exigeaient! il fallait réveiller le passé, éclairer les cinq continents, descendre au centre de la terre et tourner autour de la lune. Quand on $m$ 'astreignait à des exercices oiseux, mon esprit criait famine et je me disais que je perdais un temps précieux. J'étais frustrée et j'étais coupable : je me hâtais d'en finir toute consigne se brisait contre mon impatience. ",3

Les études lui ont permis de témoigner de son développement, Simone de Beauvoir a pu avancer dans l'existence, cet objectif l'a poussée à terminer son cursus scolaire avec une année de moins. Elle était la meilleure élève au cours Désir. Elle voulait sortir du même destin que les femmes de la bourgeoisie française des années vingt, L'intérêt pour l'éducation a conduit Simone de Beauvoir à trouver le but suivant, elle a décidé d'être professeur. La fonction de professeur a garanti l'indépendance financière et la progression des esprits à travers la transmission de ses connaissances :

${ }^{1}$ Mémoires d'une jeune fille rangée, Ibid, p, 23.

${ }^{2}$ BEAUVOIR (Simone de), Pour une morale de l'ambiguité, Gallimard, France, 2008, p. 160.

${ }^{3}$ Mémoires d'une jeune fille rangée, Ibid, p. 92 
" Je pensais à moi du dedans comme une personne en train de se faire, et j'avais l'ambition de progresser à l'infini, l'Élu, je le voyais du dedans comme une personne achevée... "'

Nawal El-Saadawi n'a pas indiqué sa formation intellectuelle, mais elle a raconté plusieurs expériences qu'elle a vécues à la famille, au village surtout qu'elle a travaillé comme pneumologue dans de différents endroits à la campagne et au Caire. Elle a signalé que l'enseignement ne lui a pas donné le savoir et que la lecture et l'écriture ont joué un grand rôle dans sa vie.

D'ailleurs, Simone de Beauvoir renonce à l'amour, devenir intellectuelle, Elle disposait également d'une volonté de l'ascension intellectuelle, elle s'adonne à ses travaux, elle a une vision bien étonnante du bonheur, celui-ci ne provient pas des livres et la littérature était par définition une entreprise solitaire. Pour l'écrivaine française, être intellectuel consiste à pouvoir découvrir la contradiction. Simone de Beauvoir a connu des conditions familiales qui ont contribué à sa formation en tant qu'intellectuelle. La capacité de découvrir le déséquilibre a conduit Simone de Beauvoir à devenir intellectuelle :

"L'individualisme de papa et son éthique profane contrastaient avec la sévère morale que m'enseignait ma mère. Ce déséquilibre qui me vouait à la contestation explique en grande partie que je sois devenue une intellectuelle. $»^{2}$

Elle voit que l'intellectuel doit se situer toujours à gauche et refuser les hiérarchies sociales, la notion d'élite et la défense des intérêts de classe. L'intellectuel s'occupe de la vérité, il fait preuve d'esprit de contestation, il possède un projet, une pensée et une œuvre. Il doit agir sur le monde comme témoin ou comme acteur. Pour elle, l'intellectuel doit avoir la création qui constitue la compétence de lier les choses les unes aux autres. Simone de Beauvoir avait une tendance à l'universel, sa famille a observé l'intérêt de Simone de Beauvoir pour de nombreuses sciences " Simone s'intéresse à tout. ", Elle s'intéressait à plusieurs domaines : l'astronomie, l'archéologie, la paléontologie,

\section{3-3- La révolte}

Selon Julia Kristeva, la révolte signifie une situation politique prise contre un régime “ révolte et révolter ... impliquent d'emblée un détournement qui sera

${ }^{1}$ Mémoires d'une jeune fille rangée, Ibid. p. 192.

${ }^{2}$ Mémoires d'une jeune fille rangée, Ibid, p. 57

${ }^{3}$ Ibid, p. 150. 
très tôt assimilé à un rejet de l'autorité". Kristeva ajoute plus de détails révélateurs : " dès 1501 est attesté le sens de " retournement d'allégeance " passage à l'ennemi ou abjuration religieuse, proche de l'Italianisme " volteface'. Elle mentionne un sens psychologique : " le mot comprend une idée de violence et d'excès par rapport à une norme, et correspond à " émouvoir ", d'où " émeute " pour révolte". ${ }^{1}$

La révolte de Nawal El-Saadawi est apparue dans la forme et le fonds de ses autobiographies, l'intimiste égyptienne est partie du refus de son statut particulier et de la communauté opprimant les femmes, elle est arrivée à rêver de la chute du régime et du changement du monde, elle a voulu détruire le monde existant d'un côté, triompher de l'obéissance et de la fidélité d'un autre côté. Pour elle, ces deux termes qui se lie à l'amour, reviennent à l'ère de l'esclavage, en conséquence, l'amour lui signifie la servitude, celle-ci implique l'obéissance et la fidélité. Lorsque la petite Nawal El-Saadawi posait la question suivante : pourquoi les femmes mariées souffrent-elles ? Les femmes adultes lui répondaient que tout provient de Dieu, c'est pour cette raison, Nawal El-Saadawi a lié lors de son enfance l'origine des malheurs des femmes aux mœurs sociaux et interprétations religieuses à force d'entendre de la part des femmes adultes la répétition des trois termes: Dieu, malheurs et mariage " Depuis l'âge de 6 ans, je répète par cour ces trois mots, je les prononce d'un seul souffle : ' Dieu, malheurs, mariage ". ${ }^{2}$ Par suite, elle fuyait le mariage au cours de son adolescence.

Nawal El-Saadawi voit que les femmes avaient historiquement et biologiquement la supériorité aux hommes, elle a consacré un livre intitulé, Alonsa hia Alasl (la femelle est l'origine) où elle a mené les preuves de la suprématie féminine, elle trouve dans la procréation et la maternité un point fort, pour elle, les femmes donnent la vie, elles méritent au moins d'obtenir l'égalité des sexes, la révolte de Nawal El-Saadawi contre la condition féminine se tourne en une éclatante revanche sur tous les hommes.

Somme toute, Nawal El-Saadawi défendait son existence contre la société antiféministe, elle se voyait une femme de libre esprit et une femme révoltée. Ce

${ }^{1}$ Sens et non-sens de la révolte, pouvoirs et limites de la psychanalyse 1 , Ibid, pp. 10,11 .

2 Texte intégral :

"منذ السادة من عمري و أنا احفظ هذه الكلمات الثناث عن ظهر قلب في عبارة واحدة:" ربنا المصائب الجواز."

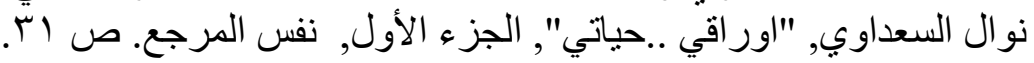


type de femmes est refusé par sa société qui n'accepte que les femmes faibles et soumises à l'homme.

“ Ma mère m'a enfantée dans ce monde qui n'est pas ma maison, la terre n'est pas la mienne, le ciel n'est pas le mien, la famille n'est pas la mienne, je n'ai ni terre, ni ciel, ni famille?','

Tandis que Simone de Beauvoir a développé nettement le statut féminin inférieur dans Le Deuxième sexe publié en 1949 en deux volumes : le premier volume a parlé de l'histoire de la condition féminine, le deuxième a traité la formation des femmes, elle a résumé toute sa pensée dans une phrase très célèbre : «on ne nait pas femme on le devient. » ${ }^{2}$ Il s'agit que la femme est un produit social, culturel et historique et que la notion de la nature féminine n'existe pas. Simone de Beauvoir voit que les femmes ne peuvent pas travailler comme les hommes à cause de leurs fonctions naturelles, ces dernières désignent la conception, la reproduction, la maternité et les règles, elles constituent selon l'écrivaine française un point faible, mais elles ne doivent pas légitimer l'inégalité des sexes.

L'écrivaine française rejetait tous les ordres injustifiés des autres : 'Quand on prétendait m'imposer des contraintes injustifiées, je me révoltais. ${ }^{, 3}$ Elle a saisi la différence intellectuelle entre elle et son père à propos des goûts littéraires, elle refusait sa conception du mariage qui se rapporte sur l'autorité du mari sur la femme et accepte le droit du mari de " donner des coups de canif dans le contrat ", , de même, elle était choquée par un fait divers sur l'avortement qui était considéré comme un délit parce que : " ce qui se passait dans mon corps ne concernait que moi ; aucun argument ne m'en fit démordre. " ${ }^{5}$ les opinions de son père sont provenues de la culture bourgeoise en cours, c'est pourquoi, elle s'y opposait violemment : ' Je refusais les hiérarchies, les valeurs, les cérémonies par lesquelles l'élite se distingue..." Sa critique a consisté à liquider la bourgeoisie en faveur de toute la société : elle était dupe de sa conviction précédente qui reposait sur les intérêts de la bourgeoisie s'accordant avec ceux de

\section{${ }^{1}$ Texte intégral :}

“ولاتني أمي في هذا العالم. هذا العالم ليس بيتي. الإرض ليست أرضي. السماء ليست سمائي. الاهل ليسوا أهلي.

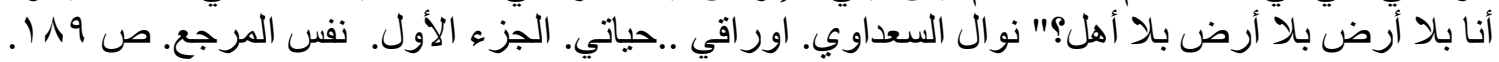

2 BEAUVOIR (Simone de), Le Deuxième sexe II, Gallimard, France, 1976, p. 13.

${ }^{3}$ Mémoires d'une jeune fille rangée, Ibid, p. 31.

${ }^{4}$ Ibid, p. 249.

${ }^{5}$ Id. 
l'humanité, “ en tout cas, j'étais victime d'une injustice et peu à peu ma rancune se tourne en une révolte." Elle a décidé de se séparer totalement de sa classe. $\mathrm{Ne}$ pas trouver sa place la voue à l'Inquiétude.

Nawal El-Saadawi a saisi le sens de sa vie, telle est la tentative de se libérer de toutes les contraintes sociales et intellectuelles, c'est pourquoi elle a répété ses rêves d'être tantôt un poisson, tantôt un oiseau :

" mon inconscient se réveillait, bougeait dans ma tête, il me fait voler vers le ciel, me fait plonger à la mer, me fait couler au fond de la terre et mourir à l'intérieur de la tombe. ",2

Nawal El-Saadawi répudie la peur de Dieu qui l'a transformée lors de son adolescence en personne lâche qui faisait l'ablution et priait tout le temps par peur de Dieu. Elle a rejeté la période où elle a perdu la volonté de découvrir les contradictions quand elle a lu le Coran et l'histoire du prophète qu'elle apprenait à l'école, elle a regretté la transformation intellectuelle qui s'est passée à cette période, c'est à dire, une enfant sceptique de la justice de Dieu est devenue une jeune fille très croyante. Ses souvenirs enfantins constituent aux yeux de l'adolescente un péché qu'elle devait se racheter. Elle incarne la fille idéale mûre. En résumé, elle a affirmé que la société a défiguré l'esprit pur de l'enfant par ses mœurs et ses contraintes, c'est pour cette raison, l'auteure égyptienne a préféré retourner à l'état innocent de l'enfant.

Quant à Simone de Beauvoir, la situation familiale a représenté l'origine de la future incroyance de Simone de Beauvoir et sa désaffection religieuse. Elle n'a pas ensuite accepté selon la foi catholique le concept du déni ou du renoncement aux tentations de la chair et aux biens de ce monde en vue d'un salut céleste. Simone de Beauvoir opte pour la vie d'ici-bas et pour ses plaisirs physiques. Donc elle a perdu sa croyance en Dieu, cette perte de foi n'a pas eu lieu rapidement, elle a cherché le remplaçant de Dieu, elle croyait aux mœurs sociales qui se remplissent de valeurs chrétiennes que son professeur de littérature française Garric lui a enseigné à l'institut Sainte Marie, elle a renoncé au siècle, elle cherchait les expériences mystiques désordonnées, elle a eu recours à la tentation de la débauche, forme inversée de la quête mystique désordonnée, mais

${ }^{I}$ Mémoires d'une jeune fille rangée, Ibid, p. 150.

2 Texte intégral :

$$
\begin{aligned}
& \text { "عقلي الباطن كان يصحو في النوم, يتحرك داخل رأسي, يجعلنا اطير واحلق في السماء في جوف البحر, في }
\end{aligned}
$$

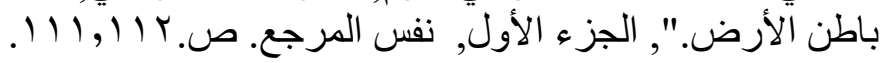


ses tentatives ont échoué, enfin, elle a adopté le pari terrestre au détriment du pari céleste :

"J'avais cessé de croire en découvrant que Dieu n'exerçait aucune influence sur mes conduites : elles ne changèrent donc pas lorsque je renonçai à lui. » ${ }^{1}$

La prise de conscience de l'absence de Dieu a contribué à adopter la perspective existentialiste et à construire une morale authentique, l'individu sans Dieu affirme sa valeur et la maitrise de son destin. Lorsque Simone de Beauvoir a envisagé des crises de désespoir et d'angoisse, elle a déterminé le but de sa vie, telle était la libération définitive de toutes les contraintes religieuses afin de poursuivre son ascension qui a constitué le sens de sa vie :

"Toutes mes journées avaient désormais un sens : elles m'acheminaient vers la libération définitive. $»^{2}$

Nous pouvons trouver dans Mémoires d'une jeune fille rangée le sens de conversion mais en sens inverse il s'agit d'un anti-récit de confession parce que Simone de Beauvoir y a raconté la transformation des valeurs concernant sa religion. Dès son enfance, elle accompagnait sa mère au catéchisme, en plus elle recevait une éducation et des règles un peu rigide au Cours Désir. Elle s'est déplacée au mysticisme qui affirme la présence divine sensible au cœur, mais, il $\mathrm{y}$ avait des sujets qui l'inquiétaient comme le thème de la naissance que personne ne lui a donné la réponse correcte en disant que les parents achètent leurs enfants, peu à peu elle n'a pas pu croire à cette image, elle a accepté l'interprétation religieuse malgré son ambiguïté :

"C'est Dieu qui crée les enfants ", Il avait tiré la terre du chaos ... le recours à la volonté divine tranquillisait ma curiosité : en gros, elle expliquait tout. ",3

D'autre part, Nawal El-Saadawi a détesté les riches comme son père qui disait qu'ils sont un soulier plein d'argent. Elle avait une inquiétude à cause de sa pauvreté, mais son père soulageait ce sentiment. C'est pourquoi l'autobiographe égyptienne se voyait immunisée contre le luxe et elle a hérité de son père le mépris pour les riches. Elle vivait pendant ses études secondaires au Caire, chez son oncle dans un petit appartement situé dans un immeuble en mauvais état. La maison de son grand-père maternel était triste, cette personne était militaire et il imposait un système strict à la maison, il s'agit du patriarcat héritier de la

${ }^{1}$ Mémoires d'une jeune fille rangée, Ibid, p. 182.

2 Mémoires d'une jeune fille rangée, Ibid. p.373.

${ }^{3}$ Ibid, p. 29. 
bourgeoisie, ce patriarcat détruit tout. Nawal El-Saadawi était entourée des femmes de la famille opprimées telles : sa mère, ses grands-mères, ses tantes, les époux étaient oppresseurs et dictateurs comme les pères et les grands-pères. Elle avait bon espoir de changer le monde. Elle trouvait que la femme devait s'armer de la science et du pouvoir économique, que le divorce désignait la libération de toutes les contraintes. La corruption inquiète Nawal El-Saadawi comme le pistonnage qui régnait en Egypte dans les années quarante surtout quand elle était à la faculté de la médecine, elle avait du mal à entrer chez le doyen de la faculté pour obtenir une bourse d'études destinée aux privilégiés, le directeur de son bureau a demandé si elle avait eu une carte de recommandation pour rencontrer le doyen, elle s'est mise en colère contre cette question, elle a pu surmonter sa peur causée par la modestie de sa famille, elle a ouvert la porte de force et elle a réussi à obtenir l'approbation du doyen.

Simone de Beauvoir a décrit son milieu avec peu d'interventions. Malgré les moments heureux de l'enfance, elle n'était pas fascinée par cette phase puisqu'elle était trompée par les valeurs bourgeoises capitalistes de son milieu qui fondent les classes sociales et qui justifient la discrimination entre les pauvres et les riches d'une part, les hommes et les femmes d'autre part, l'autobiographe française refusait les hiérarchies, les valeurs, les cérémonies par lesquelles l'élite se distinguait. Sa critique consiste à liquider la bourgeoisie en faveur de toute la société : “en tout cas, j'étais victime d'une injustice et peu à peu ma rancune se tourne en une révolte." Elle a décidé de se séparer totalement de sa classe. $\mathrm{Ne}$ pas trouver sa place la voue à l'inquiétude :

\section{3-4- La mission d'écrire}

Selon Chikweney Okonjo, dans un livre intitulé, Querraat fi Amal Nawal El-Saadawi ${ }^{2}$ (Lectures dans les œuvres de Nawal El-Saadawi), l'autobiographie représente pour son écrivain un traitement et une libération, elle lui donne le sentiment de la vérification et de l'accomplissement.

La mission d'écrire qui a constitué le dernier sens de leur vie, occupait une grande place dans leur existence, les deux écrivaines cultivaient différentes sortes de l'art : l'écriture et l'interprétation. Elles aimaient la lecture dans de nombreux domaines. Il faut souligner que Mémoires d'une jeune fille rangée et les écrits

${ }^{1}$ Mémoires d'une jeune fille rangée, Ibid, p. 150.

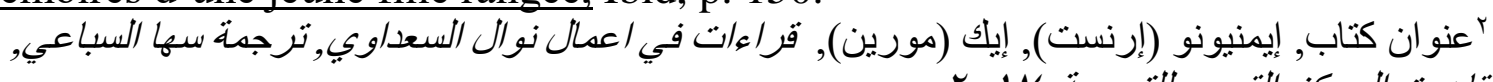


autobiographiques de Nawal El-Saadawi ${ }^{1}$ font partie du récit de vocation où elles ont raconté les premières tentatives d'écrire.

Nawal El-Saadawi était exilée aux Etats-Unis à cause de ses écrits courageux, Elle vivait un état d'ambiguïté entre la tristesse du présent et le bonheur du passé, elle était à cheval, elle souffrait d'un déséquilibre, le seul moyen de la libérer de ce sentiment, c'est l'écriture qui défie le temps présent, elle sert à être le port conduisant l'auteur au passé, elle dégage les souvenirs passés de l'oubli ou de la mort. Elle essaie de récupérer des parties de sa vie qui sont tombées dans le néant et de les retirer des griffes du passé, de sauver les moments qui s'enfuient et se cachent loin de la mémoire et des yeux des gens, ce sont les moments de la douleur, du désespoir, de l'affaiblissement et du déclin A travers l'écriture, elle tente de récupérer le visage de sa mère qui disparait parfois de la mémoire et qui apparaît des fois. Les papiers jouent un grand rôle dans la vie de Nawal El-Saadawi, ils étaient sa vie et le rêve de son enfance et de sa jeunesse bien qu'ils portent ses souvenirs douloureux et l'histoire de son amour, l'écrivain les aimait, elles les récupèrent dans son imagination. Elle découvre le caché : A travers l'écriture, elle voit les visages derrière les brumes, les mers et l'océan, le temps en abime et l'esprit. Mais, elle trouve que le fait d'utiliser l'esprit constitue un crime pour la société égyptienne.

Nawal El-Saadawi a écrit des romans et des pièces de théâtre pendant ses études, elle se passionne pour l'art, son frère Talaat et elle avaient organisé au rez-de-chaussée de leur immeuble un spectacle des marionnettes composé par l'écrivaine. Leurs parents, leurs proches, leurs amis y ont assisté et ils l'ont admirée,

La rencontre de Simone de Beauvoir avec Sartre est un point décisif dans sa vie, celui-ci lui inspire la consécration suprême de tous les mandats précédents, il s'agit de l'adaptation au monde réel et la mission d'écrire qui lui remporte l'éternité : "Notre mémorialiste tâtonne " dans son existence et le sens lui en échappe. ",

Pour Simone de Beauvoir, l'écriture comble le vide laissé par l'absence de Dieu, l'intimiste française a signalé sa vision de l'écriture dans sa vie par rapport à Sartre : "Je m'étais crue exceptionnelle parce que je ne concevais pas de vivre

${ }^{1}$ Nawal El-Saadawi a publié son autobiographie en cinq œuvres, aoraki hayati (اور/قي.. حياتي) en trois volumes, Mémoires de la prison des femmes, Rahalati fi al-alam (رحالتي في العالم).

2 Mémoires d'une jeune fille rangée, Ibid, p. 63. 
sans écrire : il ne vivait que pour écrire. ${ }^{1}$ L'écriture lui était une question de choix, elle n'a rien à voir avec la question du don ou de la révélation d'un souffle divin. Elle essaie toujours de réaliser tout à travers l'imagination. Elle cherche dans l'écriture une sollicitude, l'écriture prend une vie autonome.

Simone de Beauvoir a commencé à inventer le jeu pour Poupette, cela renforce le goût du récit et son aptitude à la communication, elle a rédigé au cours Désir des compositions françaises qui constituent une véritable création, malgré les dénégations ultérieures de la narratrice : elle surestime certaines valeurs :

«En tout cas, je devais préserver ce qu'il y avait de plus estimable en moi : mon goût de la liberté, mon amour de la vie, ma curiosité, ma volonté d'écrire. " ${ }^{2}$

Pour Simone de Beauvoir, la littérature jouait un rôle considérable lors de son enfance, elle lui a donné la capacité de venger la réalité en l'asservissant à la fiction. La littérature exige un univers solitaire, l'écriture à laquelle Simone de Beauvoir s'intéressait, apportait à ses problèmes une solution privilégiée.

Enfin, l'écriture représente pour les deux écrivaines un acte de révolte et de création contre une société déséquilibrée. Le projet de Simone de Beauvoir vise à concilier ses goûts, ses valeurs, ses ambitions, sa condition de femme et son statut d'être mortel. Pour Nawal El-Saadawi, elle a la volonté de reconstruire le monde sur l'égalité et la liberté.

\section{$\underline{\text { La conclusion }}$}

Après une profonde lecture, on a découvert que les deux écrivaines ont parlé des mêmes questions mais elles ont donné des interprétations. A propos des résultats de notre analyse qui peuvent répondre à la problématique de cette étude, on a trouvé un rapport entre l'écriture de soi et le sens, le premier concept exige le don d'un sens à sa vie en faisant son récit rétrospectif, il s'agit d'une nouvelle interprétation qui a un rapprochement d'une signification, tel est l'un des sens du deuxième concept. Pour la deuxième question concernant la signification du mot « quête », celui-ci comprend simplement la recherche, mais il implique d'après notre investigation la transformation et le changement parce que les deux écrivaines ont offert plusieurs sens de leur vie correspondant aux événements et aux expériences vécues. Quant à la troisième question, les deux écrivaines ont atteint un sens définitif de leur vie, telle est la libération de toutes les contraintes et de toutes les restrictions de ce monde dominé par le capitalisme bourgeois pour

${ }^{1}$ Ibid, p. 448.

2 Id. 
réaliser l'égalité, les deux femmes ont lutté contre les valeurs bourgeoises qui tyrannisent à la fois les pauvres et les femmes en faveur d'une petite minorité capitaliste monopolisant l'autorité politique et l'argent. Mais Nawal El-Saadawi était plus atroce que Simone de Beauvoir en ce qui concerne la critique de cette classe sociale en général et des hommes en particulier.

En bref, les deux écrivaines ont réclamé la liberté et l'égalité entre les deux sexes et la reconnaissance des femmes par leur société. 


\section{Bibliographie}

\section{Corpus et Ouvrages en français}

DE BEAUVOIR (Simone) :

Le Deuxième sexe, 2 volumes, Paris, Gallimard, 1976.

Tout compte fait. Paris, Gallimard, 1984.

La force de l'âge, Paris, Gallimard, 1986.

Mémoires d'une jeune fille rangée. Paris, Gallimard, 2008.

Pour une morale de l'ambiguïté, Gallimard, France, 2008

ETIEMBLE, Comparaison n'est pas raison, Paris Gallimard, 1963.

GENETTE (Gérard), Figures III. Paris VI ${ }^{\mathrm{e}}$, Seuil, 1987.

KRISTEVA (Julia), Sens et non-sens de la révolte, pouvoirs et limites de la psychanalyse 1, Paris, France, 1996.

\section{LEJEUNE (Philippe) :}

Le pacte autobiographique 1. Paris VI ${ }^{\text {e }}$. Seuil. 1975.

L'autobiographie en France, $2^{\mathrm{e}}$ éd, Paris, Armand Colin, 2004.

MIRAUX (Jean-Philippe), l'autobiographie, écriture de soi et sincérité, Paris, Nathan, 1996.

PICHOIS (Claude) et ROUSSEAU (André-Michel), La littérature comparée,

Paris, Armand Colin, 1967.

REY (Alain), le dictionnaire le Petit Robert, France, 2014.

\section{Thèses}

HAZAN (Isabelle), Simone de Beauvoir, mémorialiste, Université Paris X Nanterre, 1996-1997 (Maitrise de Littérature moderne)

\section{Sitographie}

https://journals.openedition.org/insaniyat/16591

https://cutt.us/bqcpN

https://theses.univ-

lyon2.fr/documents/getpart.php?id=lyon2.2006.cisse_m\&part=109998 


\section{مصادر ومراجع باللغة العربية}

نوال السعداوي,

$$
\begin{aligned}
& \text { "اور اقي ..حياتي", الجزء الأول, مكتبة مدبولي, الطبعة الثاني, ج . . ؟ . } \\
& \text { " اور اقيى ..حياتي", الجزء الثاني, مكتبة مدبولي, الطبعة الثاني, ج . . Y. } \\
& \text { " اور اقيى ..حياتي", الجزء الثالث, مكتبة مدبولي, الطبعة الثاني, ج ـ . ؟ . }
\end{aligned}
$$

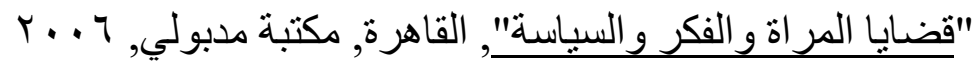

$$
\begin{aligned}
& \text { "الانثى هي الأصل", كتب عربية، مصر, ع } 9 \text { أ. } \\
& \text { إيمنيونو (إرنست), إيك (مورين), قراءات في اعمال نوال السعداوي,ترجمة سها السباعي, القاهرة, }
\end{aligned}
$$

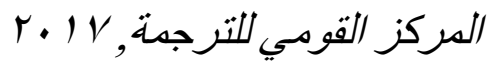

سارتر (جون بول), "الوجودية مذهب إنساني", تعريب عبدالمنعم الحفني, الطبعة الأولى, مطبعة الدار

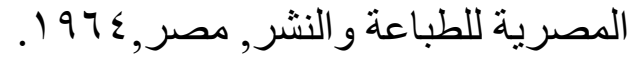

ماي (جورج), "السيرة الذاتية", تعريب محمد القاضي و عبد الله صول, بيت الحكمة, قرطاج, 999 ا. 


\section{البحث عن معنى فى كتابة الذات عند نوال السعداوى وسيمون دو بوفوار رمضان حمدان محمد \\ قسم اللغة الفرنسية- كلية الآداب-جامعة حلوان}

الملخص

تهنم دراستنا بالتعبير عن الوضع الإنساني بما يتضمنه من عملية البحث عن معنى في عالم يفتقد للمعنى, عالم

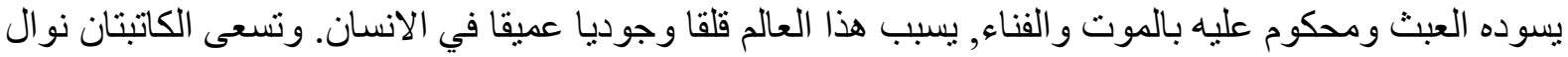

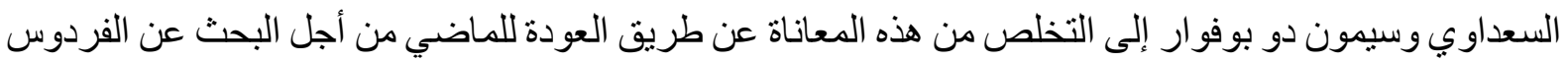

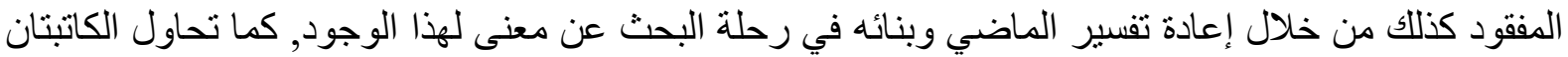

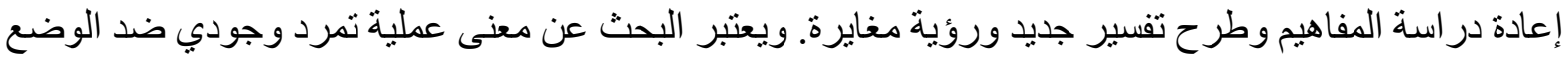

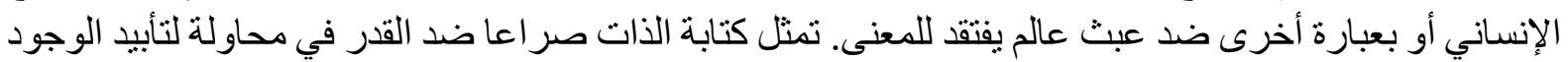

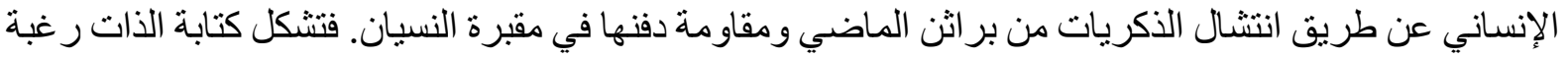

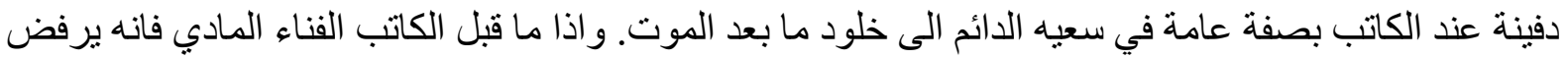
الفناء تماما المعنوي والقلق و الالم الذي يحملهم بداخله. ونجد هذا الثكل من التمرد عند الكاتبتين. الكلمات المفتاحية:تمرد؛ بحث؛ شرط انساني؛ معاناة؛ عبث 